\title{
Epifluorescent imaging study of the effect of anti-diabetic drug metformin on colorectal cancer cell lines in vitro
}

\author{
Priyadharshini Venkatasubramani ${ }^{1,2, *}$, Chao Sima ${ }^{1,2}$, Jianping Hua ${ }^{1,2}$, Milana Cypert ${ }^{2}$, Michael Bittner ${ }^{1,2}$ and Aniruddha Datta ${ }^{1,2}$ \\ 1 Electrical and Computer Engineering Department, Texas A\&M University, College Station, Texas, 77840, USA \\ 2 TEES-AgriLife Center for Bioinformatics and Genomic Systems Engineering (CBGSE), College Station, Texas, 77845, USA
}

\begin{abstract}
Metformin, a widely used anti-diabetic drug, has recently been associated with inhibition of cell proliferation in multiple cancers. However, it is not clear if the reduction in proliferation on treatment with metformin is a result of cell death or slowdown in the rate of growth of cancer cells, because cell viability assays measure only the number of cells at the beginning and end of the experiment. The aim of this study is to utilize a fluorescent imaging technique to directly follow cell death overtime in order to investigate the effect of metformin on colorectal cancer cells HCT116 and SW480. Epifluorescent imaging analysis carried out using ImageXpress Micro XLS High-Content Imaging System show that there is no significant change in cell death observed in the cancer cell lines, as compared to the control, over multiple closely spaced time points, suggesting that metformin in pharmacological doses may not be an effective inducer of cell death in these colon cancer cell lines.
\end{abstract}

Keywords: high-content imaging; metformin; diabetes; colon cancer; cancer metabolism; cell death

\section{Introduction}

Metformin, an FDA-approved biguanide, is known to reduce levels of circulating glucose and is widely used for the treatment of diabetes mellitus. Epidemiological studies of cancer patients with diabetes [1] led to the initial indication that metformin may be associated with reduced cancer-related mortality. Subsequently, a number of in vitro and in vivo studies on cancer cells have reported a negative association between cancer risk and the use of metformin $[2,3]$. As a result, there has been an increasing effort to understand the mechanism of action of metformin in an attempt to reposition the drug for the treatment of cancer.

The effects of metformin on diabetic patients diagnosed with colorectal cancer was studied in [4] by means of survival analysis. Data pertaining to 595 patients diagnosed with both colorectal cancer and diabetes mellitus were analyzed, and metformin use was found to be associated with decreased colorectal cancer specific mortality. A population-based cohort study [5], also connected metformin use with reduced colon cancer risk. Consequently, metformin is being debated for use as a prophylactic agent for colon cancer.

The anti-cancer effect of metformin has in part been attributed to its up-regulation of adenosine monophosphate (AMP) activated protein kinase activity [6, 7]. Studies have suggested that the activation of AMPK by metformin further leads to the inhibition of mammalian target of rapamycin (mTOR) in breast cancer cells [8].

A study on the effect of metformin in breast cancer cell lines reported dose-dependent reduction in cell proliferation in cell lines MCF-7 and MCF-10A [7]. As per this study, metformin in concentrations of $2.5-20 \mathrm{mM}$ led to a reduction of about $40-70 \%$ in cellular proliferation of cancer cells after exposure for 72 hrs (Cell proliferation measurement in [7] was carried out by calculating percentage reduction in the intensity of Alamar Blue dye at the end of the experiment).

A follow-up of the study conducted in [7] reported that the use of metformin led to reduction in global protein synthesis and decrease in cap-dependent translation

\footnotetext{
*Corresponding author: Priyadharshini Venkatasubramani, Electrical and Computer Engineering Department, Texas A\&M University, College Station, Texas, 77840, USA. Tel.: (979) 900-4283; Email: priya26@tamu.edu

Received 17 February 2017 Revised 23 April 2017 Accepted 27 April 2017 Published 03 May 2017

Citation: Venkatasubramani P, Sima C, Hua J, Cypert M, Bittner M, Datta A Epifluorescent imaging study of the effect of anti-diabetic drug metformin on colorectal cancer cell lines in vitro. J Cancer Res Ther. 2017; 5(4):19-23. DOI: 10.14312/2052-4994.2017-4

Copyright: (c) 2017 Venkatasubramani P, et al. Published by NobleResearch Publishers. This is an open-access article distributed under the terms of the Creative Commons Attribution License, which permits unrestricted use, distribution and reproduction in any medium, provided the original author and source are credited.
} 
in mouse embryonic fibroblasts (MEFs) [8]. The major cause of these effects of metformin was reported to be its inhibition of mTOR. Exposure to metformin for $72 \mathrm{hrs}$ in doses of $20 \mathrm{mM}$ were reported to inhibit the growth of $\mathrm{TSC2}^{+/+}$(tuberous sclerosis complex 2 ) MEFs by $53 \%$ as compared to the control samples (MEFs were stained with crystal violet, which was eluted with acetic acid, and cell growth was determined by measuring light absorption levels of the supernatant from each sample at $570 \mathrm{~nm}$ ).

Many such studies correlated the use of metformin with better prognosis for cancer patients because of the observed reduction in cellular proliferation. While a number of experiments performed in these studies [7, 8] showed a reduction in the total number of cells on treatment with metformin, there is a lot of variance in the amount of reduction observed. Furthermore, it is not clear if the use of metformin killed cancer cells or retarded the growth of cells in the cancerous cell lines, since the conventional cell death assays only provide snapshots of cellular proliferation at the initial and final time points of the experiment.

In order to capture the full dynamics of the system over an extended period of time, multiple assays need to be produced under identical conditions at different time points by harvesting cells and extracting analytes at each time point from a different cell population. In order to circumvent this cumbersome procedure, a transcriptional fluorescent imaging technique may be utilized to directly follow the death of a set of cells over time as in the current study.

Fluorescent reporters have long been used for temporal studies in experimental biology in order to study transcriptional activities of cells. In this study, a stream of images of a particular population of cells was acquired from the beginning to the end of the experiment at different closely spaced time points. These images were processed to obtain time course data that contains details about cellular activities during the course of the experiment. This data was processed and visualized in order to attain a deeper understanding of the dynamics at the cellular level.

To track cell death caused by treatment with metformin, colorectal cancer cells were stained with CellTox green dye (details explained in the materials and methods section), which preferentially stains dead cell DNA when cell membrane integrity is compromised, producing a nucleus specific, bright green fluorescent signal. The fluorescent signals generated in the experiment were tracked by repeatedly capturing images of the same sites and then scoring cell life and death so as to quantify cell death at different time points over a period of $37 \mathrm{hrs}$.

\section{Materials and methods}

\section{High-content epifluorescent imaging}

In our experimental protocol, a single assay was carried out by epifluorescent imaging of a site at the bottom of a well in a 384-well plate, thus generating an image of the cells in that region ( 200 to 300 cells) bearing fluorescent reporters. The automated imaging systems easily accommodate sampling an entire 384-well plate at hourly intervals. ImageXpress Micro XLS High-Content Imaging System (Molecular Devices, LLC, Sunnyvale, California, USA) was used to capture the time lapse fluorescent image data from cells in multi-well plates.

For studying cell death, fluorescent images are taken as two-color image pairs with a blue channel image for the nuclei and a green channel image for the fluorescent CellTox Green reporter. When cell death occurs and the cell membrane collapses, the CellTox Green dye stains the dead cell DNA with fluorescent green color.

\section{Quantification of cell death from images}

Visual examination of images may provide some indication of the extent of cell death at a particular imaging site, but advanced image-processing procedures followed by efficient data summarization help in acquiring reliable estimates of cell death occurring throughout the experiment. In order to assess the amount of cell death caused by metformin, select morphological features of the cell were used to label a cell as dead or alive. Primary among these features is disruption of the cell membrane, at which time the CellTox Green dye enters the cell and binds to the DNA. Additionally, pyknosis, (condensation of chromatin in the nucleus) which can be detected on the nuclear channel of the images, is another key feature of cell death. The image-processing pipeline detailed in [9] establishes thresholds for nuclear size and intensity of the dye in the nucleus in order to detect cell membrane collapse, and these thresholds are used to count the number of dead cells in the images at various sites in the well. The percentage cell death was then calculated as: \% Cell death $=$ number of dead cells/ total number of cells $\mathrm{x}$ 100.

\section{Statistical analysis}

Percentage cell death at each imaging site for the different doses of metformin were tested for statistical significance. Statistical analysis was performed using the Students $t$-test with R software, version 3.2.2 (R Foundation for Statistical Computing, Vienna, Austria) [10]. $\mathrm{P}<0.05$ was considered to indicate a statistically significant difference.

\section{Cell lines and treatments}

Colorectal cancer cell lines HCT116 and SW480 were plated at a density of 6000 cells/well in $30 \mu \mathrm{l} /$ well of Imaging Media (IM) on one 384-well microtiter plate (Greiner Bio-One, 781 09x) pre-coated with $10 \mu \mathrm{g} / \mathrm{ml}$ Rat Tail Collagen Type I (BD Biosciences, 354249) and washed 3X with sterile 1X PBS without calcium and magnesium. IM consists of $70 \%$ M-199 (Thermo Fisher Scientific, 11825015), 30\% RPMI1640 (11875085) supplemented with 10\% FBS (16000044), 11 mM D-glucose (A2494001), 20 mM HEPES (15630080) and $20 \mathrm{mM}$ GlutaMax (35050061). Nuclei of the cells were stained with Vybrant ${ }^{\circledR}$ DyeCycle ${ }^{\text {TMV} V i o l e t ~ l i v e-c e l l ~ n u c l e a r ~}$ stain (Thermo Fisher Scientific) diluted 1:15,000. The cells were allowed to attach to the surface of the plate by incubating at $37^{\circ} \mathrm{C}$ with $5 \% \mathrm{CO}_{2}$ for $6 \mathrm{hrs}$. 
After taking images of cells at the first baseline time point before treatment, the cells were treated with $5 \mathrm{mM}, 10$ $\mathrm{mM}, 15 \mathrm{mM}$, and $20 \mathrm{mM}$ concentrations of metformin diluted in IM or left untreated as control. Images of each well were taken every hour up to 36 hrs using the HighContent Imaging System.

\section{Results \& discussions}

Metformin does not cause significant cell death in colorectal cancer cell lines in vitro

We examined the effect of metformin on cell death in colorectal cancer cell lines HCT116 and SW480 through high-content epifluorescent imaging. Figure 1 shows a continuous summarized representation of percentage cell death measured on exposure of colon cancer cell lines to metformin over a period of $37 \mathrm{hrs}$. Met5, Met10, Met15 and Met20 correspond to $5 \mathrm{mM}, 10 \mathrm{mM}, 15 \mathrm{mM}$ and $20 \mathrm{mM}$ doses of metformin, respectively. The percentage cell death values shown are means of cell death values calculated at different imaging sites for each treatment condition. As seen from Figure 1a, in cell line HCT116, an increase of about $20 \%$ in cell death was observed on average at certain time points on exposure to metformin, but continuous increase was not sustained through the experiment. Figure $1 \mathrm{~b}$ shows that relative to the control, metformin caused no significant increase in cell death in the cell line SW480.

(a) Cell death induced in HCT116 by metformin

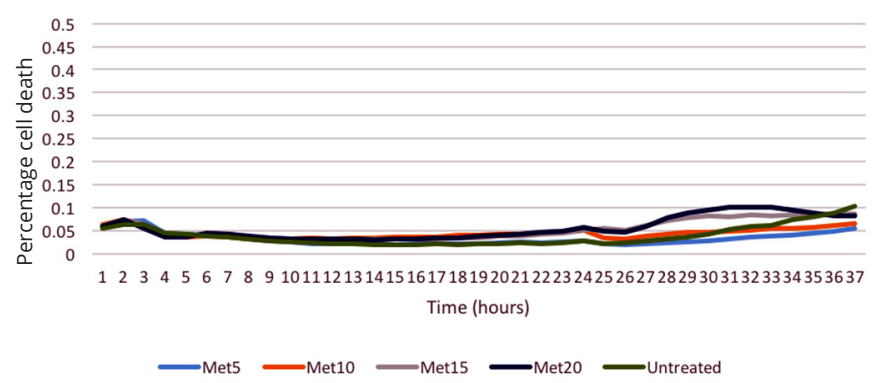

(b) Cell death induced in SW480 by metformin

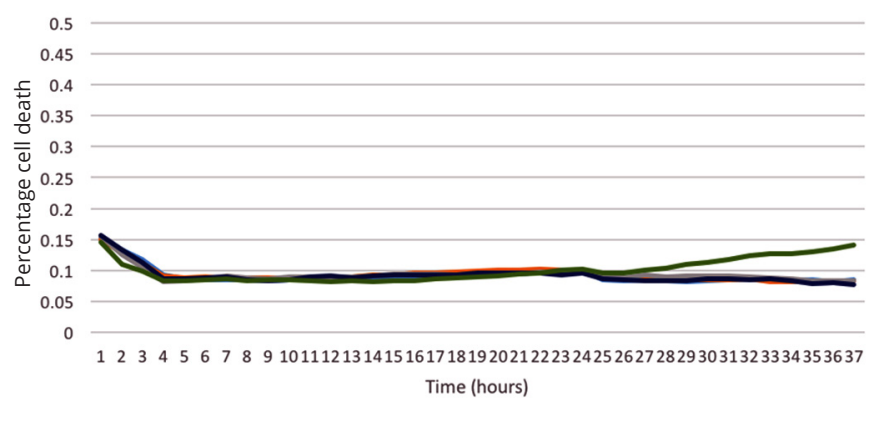

Figure 1 Percentage cell death relative to the untreated cells (control) are shown at several time points after the administration of the drug metformin in different doses to colorectal cancer cell line a) HCT116 and b) SW480. Met5, Met10, Met15 and Met20 correspond to $5 \mathrm{mM}, 10 \mathrm{mM}, 15 \mathrm{mM}$ and $20 \mathrm{mM}$ doses of metformin, respectively.

Figure 2 shows the cell death percentage at the different doses and at 4 different time points (24h, 28h, 32h, 36h) in the HCT116 cell line, relative to the control, and Figure 3 shows the cell death in the SW480 cell line.
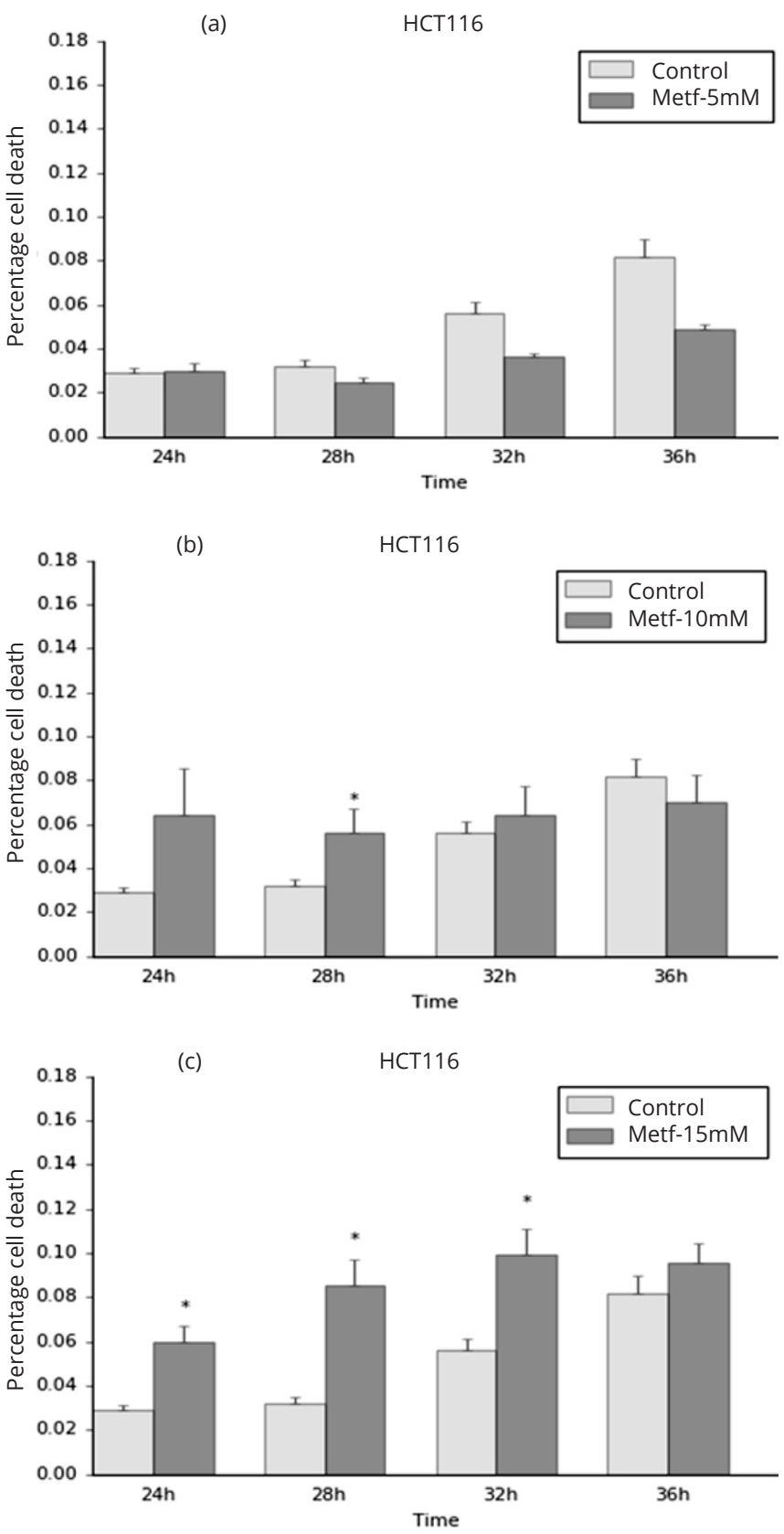

(d)

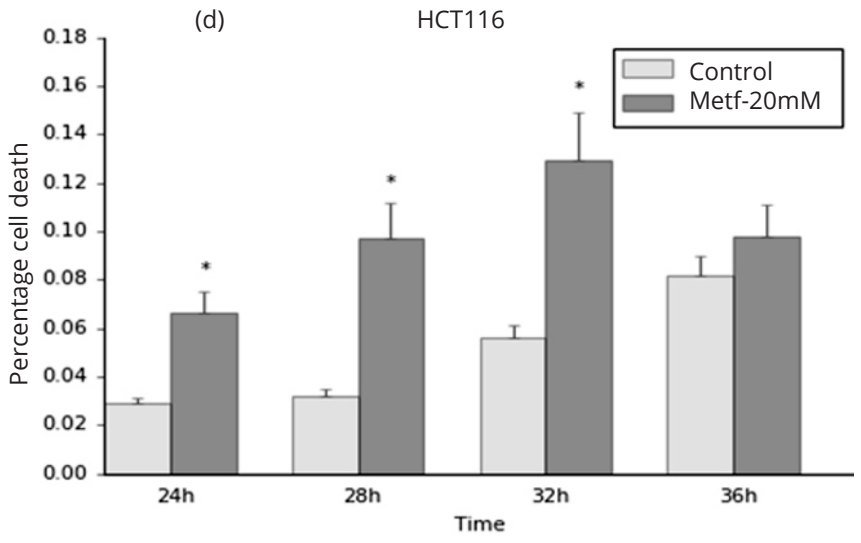

Figure $\mathbf{2 a}, \mathbf{b}, \mathbf{c}, \mathbf{d}$ Cell death induced by metformin in colorectal cancer cell line HCT116. Cells were treated with 5-20 mM metformin for $37 \mathrm{hrs}$, and percentage cell death was quantified as outlined in the Materials and Methods. Results are given as means \pm SE for 9 replicate determinations at 4 different imaging sites for each treatment at four different time points, and significant $(p<0.05)$ increase in cell death is indicated $(*)$.

Note: There were some statistically significant observations. 


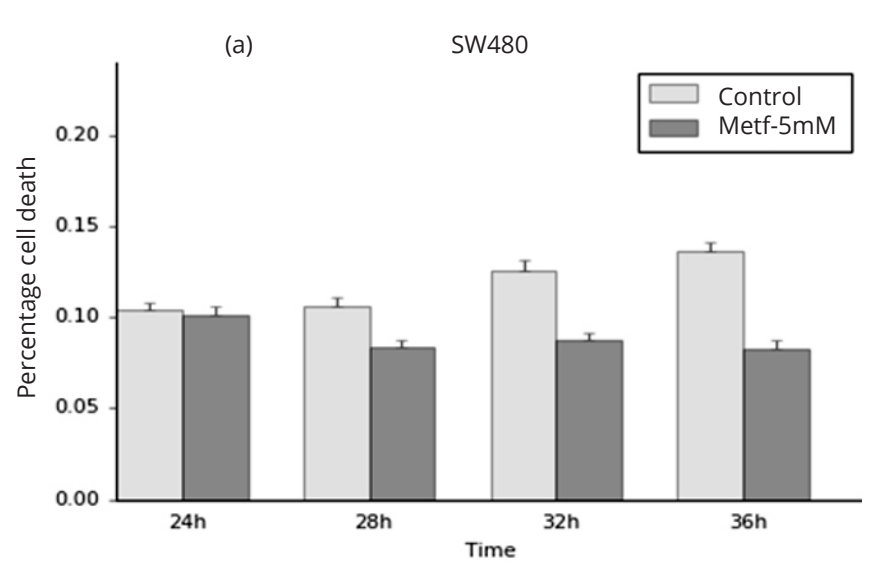

(b)

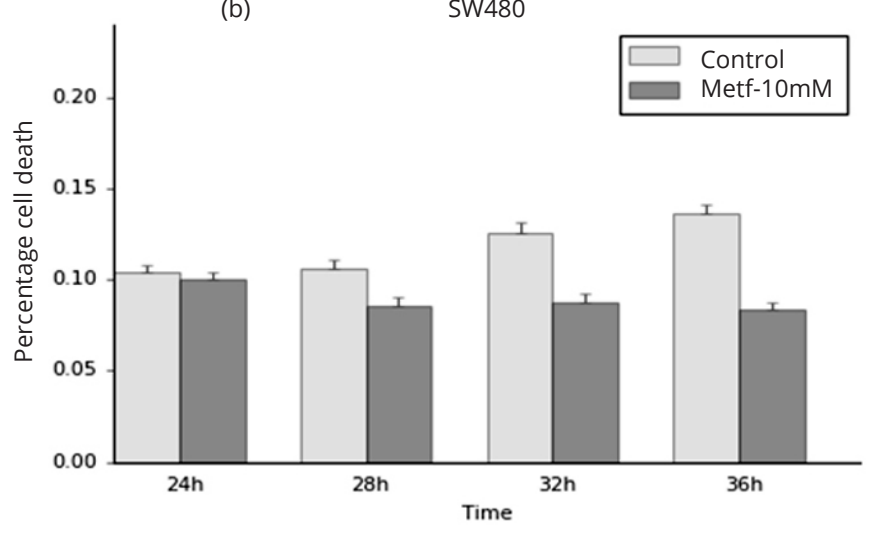

(c) SW480

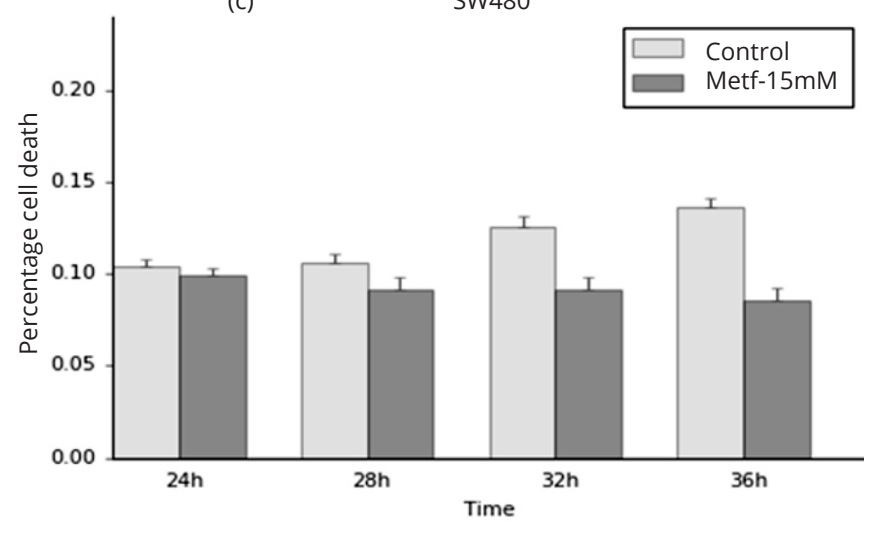

(d)

SW480

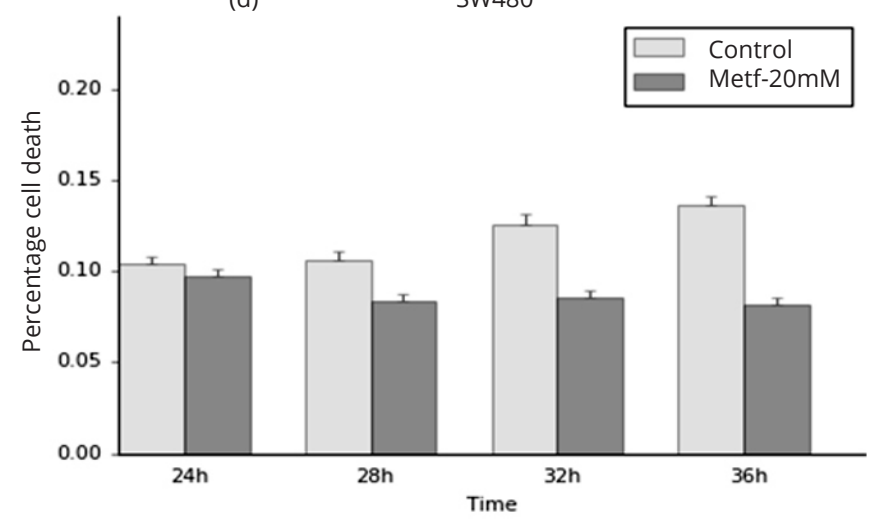

Figure $\mathbf{3 a}, \mathbf{b}, \mathbf{c}, \mathbf{d}$ Cell death induced by metformin in colorectal cancer cell line SW480. Cells were treated with 5-20 mM metformin for $37 \mathrm{hrs}$, and percentage cell death was quantified as outlined in the Materials and Methods. Results are given as means \pm SE for 9 replicate determinations at 4 different imaging sites for each treatment at four different time points, and significant $(p<0.05)$ increase in cell death is indicated $(*)$.

Note: There were no significant observations.
Drug repositioning (repurposing), a term used to refer to the identification of new applications for existing drugs, has become popular in recent years, fueled by the large scale battle against cancer and the search for anti-cancer drugs by the world-wide cancer research community. Existing drugs have already been in human use and have minimal cell toxicity, so they are appealing alternatives to discovering new drugs that might require extensive study and testing before they can be presented for FDA approval.

A potential link between metabolism and cancer has led to multiple investigations into the effects of metformin on different cancers with the ultimate goal of repositioning metformin to treat cancers. Our investigations into the action of metformin, aimed at studying the cell death caused by metformin in cancer cell lines, revealed that high concentrations of metformin caused some cell death in the cell line HCT116, which was significant up to $32 \mathrm{hrs,}$ while there was no significant cell death observed in the cell line SW480. Such inconsistent effects of metformin on cell lines has been reported before [8], where the authors observed dose-dependent effect of metformin on breast and ovarian cancer cell lines, and no effect on the HeLa cell line.

In our study, the colorectal cancer cell lines were monitored up to $37 \mathrm{hrs}$. It is possible that there may be more cell death observed if the cells are tracked for a longer time. However, the trends we observed make such a possibility unlikely. Moreover, rapid cell killing is a highly desired characteristic in a chemotherapy drug. Based on our study, metformin, when administered individually to colorectal cancer cell lines, does not seem to possess this characteristic.

The experiments carried out in our study are in vitro, where the cells are treated outside their natural environment. Consequently, intracellular signaling in the cells under study is impaired, and the responses observed may not truly represent responses from an in vivo animal study or a clinical trial. The inherent abnormality and heterogeneity of cancer tissues also make it very challenging to relate outcomes from in vitro models to human cancer outcomes. However, in vitro methods are widely established, and facilitate ease of interpretation of results since the composition of the cells being analyzed is well known beforehand. In vitro studies are also good indicators of risk and underlying biological mechanisms, and must be analyzed meticulously before proceeding to in vivo animal studies or clinical trials. The lack of clear patterns in the amount of cell death observed at different doses and time points and the large variability in the results for different cell lines in our study indicate that we need much more information about how metformin affects different cancer cell lines. It is imperative that this deficiency in understanding the effect of metformin be kept in mind when considering novel therapies based on metformin.

Interestingly, Hirsch et al. [11] found that metformin in combination with doxorubicin, a chemotherapeutic drug, inhibited the growth of cancer cells in culture in four different breast cancer cells MCF-7, MCF-10A, SKBR3 and MDA-MB-486 [11]. Additionally, in their [11] study, when 
MCF-10A cells were injected into nude mice and the tumor volume was measured at various time intervals, it was found that the combination therapy reduced the tumor volume and prevented relapse of the cancer more effectively as compared to either drug alone. The main reason for this observation was reported to be the ability of metformin to selectively kill cancer stem cells. Experimental studies [12, 13] have also indicated that metformin may potentiate the effect of certain chemotherapeutic drugs such as cisplatin, carboplatin, etc., when used in combination in the treatment of cancer cells. Our future studies will be aimed at examining the action of metformin in combination with other chemotherapy drugs.

\section{Conclusion}

The high concentrations of metformin caused some cell death in the colorectal cancer cell line HCT116, which was significant up to $32 \mathrm{hrs}$. However, there was no significant cell death observed in the colorectal cancer cell line SW480. This indicates that not all cancer cell lines respond in a similar way to treatment with metformin, and individual studies on different cell lines are necessary. A great advantage of combining a drug metformin in a chemotherapeutic cocktail with a more potent drug is that it may help in reducing the cytotoxicity of the overall dose, while maintaining the level of cell killing achieved by the standard chemotherapy drug. Hence, the utility of metformin may be in combination therapy for treating cancer, rather than individually.

\section{Funding}

This work was supported in part by the National Science Foundation under grant [ECCS-1404314] and in part by the AgriLife-TEES Center for Bioinformatic and Genomic Systems Engineering (CBGSE).

\section{Conflicts of interest}

The authors declare that they have no conflict of interest.

\section{References}

[1] Giovannucci E, Harlan DM, Archer MC, Bergenstal RM, Gapstur SM, et al. Diabetes and cancer: A consensus report. CA Cancer J Clin. 2010; 60(4):207-221

[2] Bao B, Wang Z, Ali S, Ahmad A, Azmi AS, et al. Metformin inhibits cell proliferation, migration and invasion by attenuating CSC function mediated by deregulating miRNAs in pancreatic cancer cells. Cancer Prev Res (Phila). 2012; 5(3):355-364.

[3] Kisfalvi K, Eibl G, Sinnett-Smith J, Rozengurt E. Metformin disrupts crosstalk between $\mathrm{G}$ protein-coupled receptor and insulin receptor signaling systems and inhibits pancreatic cancer growth. Cancer Res. 2009; 69(16):6539-6545.

[4] Lee JH, Kim TI, Jeon SM, Hong SP, Cheon JH, et al. The effects of metformin on the survival of colorectal cancer patients with diabetes mellitus. Int J Cancer. 2012; 131(3):752-759.

[5] Tseng $\mathrm{CH}$. Diabetes, metformin use, and colon cancer: A populationbased cohort study in Taiwan. Eur J Endocrinol. 2012; 167(3):409-416.

[6] Zhou G, Myers R, Li Y, Chen Y, Shen X, et al. Role of AMP-activated protein kinase in mechanism of metformin action. J Clin Invest.. 2001; 108(8):1167-1174.

[7] Zakikhani M, Dowling R, Fantus IG, Sonenberg N, Pollak M. Metformin is an AMP kinase-dependent growth inhibitor for breast cancer cells. Cancer Res. 2006; 66(21):10269-10273.

[8] Dowling RJ, Zakikhani M, Fantus IG, Pollak M, Sonenberg N. Metformin inhibits mammalian target of rapamycin-dependent translation initiation in breast cancer cells. Cancer Res. 2007; 67(22):1080410812
[9] Hua J, Sima C, Cypert M, Gooden GC, Shack S, et al. Tracking transcriptional activities with high-content epifluorescent imaging. J Biomed Opt. 2012; 17(4):0460081.

[10] Team RC. R: A language and environment for statistical computing. R Foundation for Statistical Computing, Vienna, Austria. 2013.

[11] Hirsch HA, Iliopoulos D, Tsichlis PN, Struhl K. Metformin selectively targets cancer stem cells, and acts together with chemotherapy to block tumor growth and prolong remission. Cancer Res. 2009; 69(19):7507-7511.

[12] Erices R, Bravo ML, Gonzalez P, Oliva B, Racordon D, et al. Metformin, at concentrations corresponding to the treatment of diabetes, potentiates the cytotoxic effects of carboplatin in cultures of ovarian cancer cells. Reprod Sci. 2013; 20(12):1433-1446.

[13] Krech T, Thiede M, Hilgenberg E, Schäfer R, Jürchott K. Characterization of AKT independent effects of the synthetic AKT inhibitors $\mathrm{SH}-5$ and SH-6 using an integrated approach combining transcriptomic profiling and signaling pathway perturbations. BMC cancer. 2010; 10(1):287. 